\title{
BMJ Open Ultra-processed foods and excessive free sugar intake in the UK: a nationally representative cross-sectional study
}

\author{
Fernanda Rauber (1D ,1,2 Maria Laura da Costa Louzada, 2,3 \\ Euridice Martinez Steele, ${ }^{1,2}$ Leandro F M de Rezende, ${ }^{2,4}$ Christopher Millett, ${ }^{2,5}$ \\ Carlos A Monteiro, ${ }^{1,2}$ Renata B Levy ${ }^{2,6}$
}

To cite: Rauber F, Louzada MLdC, Martinez Steele E, et al. Ultra-processed foods and excessive free sugar intake in the UK: a nationally representative crosssectional study. BMJ Open 2019;9:e027546. doi:10.1136/ bmjopen-2018-027546

- Prepublication history and additional material for this paper are available online. To view these files, please visit the journal online (http://dx.doi org/10.1136/bmjopen-2018027546).

Received 27 October 2018 Revised 06 September 2019 Accepted 12 September 2019

Check for updates

(c) Author(s) (or their employer(s)) 2019. Re-use permitted under CC BY-NC. No commercial re-use. See rights and permissions. Published by BMJ.

For numbered affiliations see end of article.

Correspondence to Dr Fernanda Rauber; rauber.fernanda@gmail.com

\section{ABSTRACT}

Objectives To describe dietary sources of free sugars in different age groups of the UK population considering food groups classified according to the NOVA system and to estimate the proportion of excessive free sugars that could potentially be avoided by reducing consumption of their main sources.

Design and setting Cross-sectional data from the UK National Diet and Nutrition Survey (2008-2014) were analysed. Food items collected using a 4-day food diary were classified according to the NOVA system.

Participants 9364 individuals aged 1.5 years and above. Main outcome measures Average dietary content of free sugars and proportion of individuals consuming more than $10 \%$ of total energy from free sugars.

Data analysis Poisson regression was used to estimate the associations between each of the NOVA food group and intake of free sugars. We estimated the per cent reduction in prevalence of excessive free sugar intake from eliminating ultra-processed foods and table sugar. Analyses were stratified by age group and adjusted for age, sex, ethnicity, survey year, region and equivalised household income (sterling pounds).

Results Ultra-processed foods account for $56.8 \%$ of total energy intake and $64.7 \%$ of total free sugars in the UK diet. Free sugars represent $12.4 \%$ of total energy intake, and $61.3 \%$ of the sample exceeded the recommended limit of $10 \%$ energy from free sugars. This percentage was higher among children (74.9\%) and adolescents (82.9\%). Prevalence of excessive free sugar intake increased linearly across quintiles of ultra-processed food consumption for all age groups, except among the elderly. Eliminating ultra-processed foods could potentially reduce the prevalence of excessive free sugar intake by $47 \%$. Conclusion Our findings suggest that actions to reduce the ultra-processed food consumption generally rich in free sugars could lead to substantial public health benefits.

\section{INTRODUCTION}

Excessive consumption of free sugar is associated with obesity, type 2 diabetes, dental caries and several other health outcomes. ${ }^{1-4}$ To address this associated health burden, the $\mathrm{WHO}^{5}$ recommends that free sugars should be reduced to less than $10 \%$ of total energy intake and also suggests a level below $5 \%$ to

\section{Strengths and limitations of this study}

Use of a large and nationally representative sample of the UK population, increasing generalisability.

- Use of data on free sugars rather than total sugars or sugar-sweetened beverages, which correspond to the guidelines relevant area of prioritisation.

- Use of NOVA system that has been recognised as a valid tool for public health and nutrition research and policy by international organisations.

- Dietary data obtained by food diaries are subject to potential error and bias.

- UK national dietary survey collects limited information indicative of food processing (eg, place of meals and product brands), which may lead to misclassification of food items.

obtain additional health benefits, such as reduction of dental caries. Achievement of this ambitious target will require bold and systematic efforts to reduce sugar across a variety of food products in most settings.

As defined by the NOVA food classification system, ultra-processed foods are industrial formulations of many ingredients, mostly of exclusive industrial use, that result from a sequence of industrial processes (hence ultra-processed) ${ }^{6}$ In some high-income countries, including the UK, ultra-processed foods account for more than half of total dietary energy intake. ${ }^{7-9}$ Importantly, national dietary surveys conducted in high-income and middle-income countries ${ }^{8-12}$ have shown a strong and positive association between consumption of ultra-processed foods and excessive dietary added (or free) sugar intake. Free sugars include sugars added to foods by the manufacturer, cook and consumer, plus sugars naturally present in honey, syrups and fruit juices, ${ }^{5}$ while added sugars captures all free sugars, but exclude naturally occurring sugars in fruit juices. 
Free sugar intake in the UK is high, ranging from $11 \%$ to $15 \%$ of total energy intake. ${ }^{13}$ To address this, the UK has implemented a number of measures including a sugar-sweetened beverage levy in 2018. However, action on sugar-sweetened beverages alone is unlikely to reduce population level sugar intake to WHO-recommended levels. In a more recent publication, the voluntary sugar reduction programme continues being endorsed by the government, but other measures such as restriction of advertising and in-store promotions of some sugary foods are also being considered as strategies to reduce childhood obesity. ${ }^{14} \mathrm{~A}$ better understanding of the key sources of sugar intake in the UK diet is required to inform policy development. This study aims to describe the dietary sources of free sugars in different age groups of the UK population taking into account food groups classified according to the NOVA classification system and estimate the proportion of excessive free sugars that could be potentially avoided by reducing the consumption of their main dietary sources.

\section{METHODS}

\section{Data source and collection}

We used data from the National Diet and Nutrition Survey Rolling Programme (NDNS) years 1-6 (2008/20092009/2010, 2010/2011-2011/2012, 2012/2013$2013 / 2014$ ) combined, which is a cross-sectional survey of people aged 1.5 years or older. The survey was designed to be representative of the UK population and provides comprehensive information on food intake. Details of the rationale, design and methods of the survey have been described elsewhere. ${ }^{15}$ Briefly, the sample was drawn from households randomly selected from the UK Postcode Address File, a list of all UK addresses. One adult (aged 19 years and older) and one child (aged 1.5-18 years), if available, were randomly selected from each household. Only a child was selected from some households to be part of a 'child boost' to ensure approximately equal numbers of children and adults. Participants (or in the case of children $\leq 11$ years, their parent/carer) completed a 4-day food diary and participated in an interview that included data on socio-demographic status.

Participants were asked to report all foods and drinks consumed both within and outside the home. Portion sizes were estimated using household measures or weights from packaging. Once completed, diaries were checked by interviewers with respondents and missing details added to improve completeness. Diary days were randomly selected to ensure balanced representation of all days of the week. All individuals who completed 3 or 4 days of dietary recording were eligible for inclusion in the study, giving a sample size of 9374 (4738 adults and 4636 children) participants for years 1 to 6 (2008/2009 to 2013/2014) combined.

The food intake data from completed records were coded and edited using the software DINO (Diet In Nutrients Out) and food and nutrient intakes estimated using nutrient composition data from the Department of Health's Nutrient Databank, updated for each survey year. ${ }^{16}{ }^{17}$ Free sugars are defined as sugars added to foods by the manufacturer, cook or consumer, plus sugars naturally present in honey, syrups, fruit juices and fruit concentrates. ${ }^{5}$ Intakes in the UK NDNS years 1-6 were expressed as non-milk extrinsic sugars (NMES). The term NMES captures all sugars defined by the term free sugars while also including half of the sugars present in dried, stewed or canned fruit. The NMES values could be slightly higher in some cases than the free sugar values, mostly in the non-ultra-processed food group since the term free sugar does not include sugars contributed by dried and processed fruits. Based on the assumption that those definitions are sufficiently similar for assessment and monitoring purposes, ${ }^{13}$ this study used the term free sugars.

Computerised raw data files and documentation from this survey were obtained under license from the UK Data Archive (http://www.esds.ac.uk).

\section{Food classification according to processing}

We classified all recorded food items according to NOVA, a food classification system based on the nature, extent and purpose of the industrial food processing. ${ }^{6}$ This classification includes four groups: (1) unprocessed or minimally processed foods (eg, fresh, dry or frozen fruits or vegetables; grains, flours and pasta; pasteurised or power plain milk, plain yoghurt, fresh or frozen meat); (2) processed culinary ingredients (eg, table sugar, oils, butter and salt); (3) processed foods (eg, vegetables in brine, cheese, simple breads, fruits in syrup, canned fish) and (4) ultra-processed foods (eg, soft drinks, sweet or savoury packaged snacks, confectionery; packaged breads and buns; reconstituted meat products and preprepared frozen or shelf-stable dishes) (see online supplementary table S1). The detailed description of NOVA classification can be found elsewhere. ${ }^{6} 18$

All foods in NDNS are coded as food number and grouped into subsidiary food groups $(n=155)$. When possible, subsidiary food groups were directly classified according to NOVA (see online supplementary table S2). When foods within a subsidiary food group pertained to different NOVA groups $(n=52)$, it was the food codes instead of the group, which were individually classified. By doing so, we were able to classify each underlying ingredient of homemade dishes in its corresponding NOVA group. Subsidiary food groups as classified by NOVA are described in the online supplementary table S2.

Although the NDNS database was provided with most food items systematically disaggregated into their individual components, about $4 \%$ of composite food codes were still mixed dishes compiled from two or more single-ingredient food code. ${ }^{19}$ The method we adopted to disaggregate food codes has been described previously. ${ }^{19}$ Using the core sample of years 1 to 4 (2008/2009 to $2011 / 2012) \quad(n=4125)$, we estimated that composite food codes represented only $3 \%$ of total calories. In 
this case, dishes were categorised according to the main constituent ingredient. Dishes in which a main constituent ingredient was not clearly identified (eg, chicken and vegetable soup) were classified as a specific subgroup of freshly prepared dishes based on one or more unprocessed or minimally processed food (group 1). Non-caloric supplements were not included in the analyses.

\section{Covariates}

Covariates included were age (years), sex, ethnicity (White, mixed ethnic group, Black or Black British, Asian or Asian British and other race), region (England North, England Central/Midlands, England South (including London), Scotland, Wales and Northern Ireland), survey year (years 1-6) and equivalised household income (equivalised for different household sizes and composition using the McClements equivalence scale ${ }^{15}$ ). Due to the significant proportion of missing values for the equivalised household income $(12.8 \%)$, we applied multiple imputation by chained equation method based on age, sex, ethnicity, excessive free sugar intake and ultra-processed food consumption. Multiple imputation was performed 20 times, and the Monte Carlo error analysis showed good statistical reproducibility of the results. ${ }^{20}$ We used the average of estimates from each imputed data set. Sensitivity analysis was conducted comparing findings from imputed data and complete case analysis.

\section{Data analysis}

For each survey day and age group (1.5-10 years, 11-18 years, 19-64 years and $\geq 64$ years), we defined extreme total energy intake outliers as values below the 1st and above the 99th percentiles ${ }^{21}$ (see online supplementary figure S1). Based on these criteria, we excluded 10 individuals who had all days of food diary classified as outliers. In total, 9364 (4729 adults and 4635 children) participants were eligible for inclusion in the analyses and more than 91\% completed the 4-day food diary. We used the mean of all available days of food diary for each individual.

Food items were sorted into mutually exclusive food groups according to NOVA classification. We combined the group of unprocessed or minimally processed foods with the group of processed culinary ingredients, as foods belonging to these two groups are usually mixed together in culinary preparations and, therefore, consumed together. Thus, we performed the analyses considering three groups of foods: unprocessed or minimally processed foods and processed culinary ingredients (individuals are able to determine the amount of table sugars they add), processed foods (sugar added by the food industry) and ultra-processed foods (sugar added by the food industry).

First, we estimated the distribution of total energy and free sugar intake according to the food groups. Then, we calculated the mean free sugar intake of the overall diet and the prevalence of excessive intake of free sugars. We used the WHO recommendations ${ }^{5}$ to assess the excessive intake of free sugars ( $\geq 10 \%$ of total energy). Analyses using the UK recommendations to further limit free sugar intake to less than $5 \%$ of total energy intake are presented in a supplementary table (online supplementary table S3). Analyses were carried out for the entire population and also stratified by age group.

Next, the prevalence of excessive intake of free sugars $(\geq 10 \%$ of total energy) was compared across quintiles of the energy share provided by each of the three food groups. Poisson regression was used to estimate prevalence ratios (PRs) and 95\% CIs for the associations between each of the three NOVA food group quintiles and prevalence of individuals consuming more than $10 \%$ of total energy from free sugars. Tests of linear trend were performed to evaluate the quintiles as a single continuous variable. All analyses were stratified by age group. Multiple regression models were also performed to adjust for age, sex, ethnicity, region, survey year and equivalised household income (sterling pounds). Analyses using the entire population are presented in a supplementary table (online supplementary table S4). We also evaluated the extent to which the association between the exposure (dietary contribution of NOVA food groups) and the dietary content in free sugars changed according to the survey year, by including a multiplicative interaction term (survey year×dietary contribution of NOVA food groups) in the fully adjusted models.

Finally, we estimated the proportion of excessive free sugar intake that could be potentially avoided if exposure to the risk factors was eliminated (theoretical minimum risk exposure level scenarios).$^{22} 23$ The counterfactual scenarios were defined considering the main dietary sources of free sugars. The first counterfactual scenario assumed no consumption of ultra-processed food (potentially hidden sugars), while in the second scenario table sugar consumption was set to zero. Table sugar included honey, molasses, maple syrup $(100 \%)$ and sugar added to coffee/juice and homemade dishes (potentially sugar that can be measured by the consumer). Examples of homemade dishes include biscuits, fruit pies, buns, cakes and pastries, cereal-based milk puddings and sponge pudding (see online supplementary table S2).

In both scenarios, we first calculated the prevalence of excessive free sugar intake in the UK population $\left(\mathrm{P}_{\text {popu- }}\right.$ lation $)$. We then estimated the predicted prevalence of excessive free sugar intake that would be expected had the consumption of each of these main sources of free sugars being zero $\left(\mathrm{P}_{\text {non-exposed }}\right)$. Lastly, we calculated the proportion of excessive free sugar intake that could be potentially avoided in each scenario using the following formula: $\left(\mathrm{P}_{\text {population }}-\mathrm{P}_{\text {non-exposed }}\right) / \mathrm{P}_{\text {population }}$. Prevalences were adjusted for sex, age, ethnicity, region, survey year and household income. To test more feasible scenarios, we also estimated the per cent reduction in prevalence of excessive free sugar intake from reducing the consumption of ultra-processed foods and table sugar by $50 \%$ (see online supplementary figure S2).

NDNS study weights were used in all analyses to account for sampling and non-response error. All statistical 
analyses were carried out using Stata Statistical Software V.14. The $\mathrm{p}$ values reported were two-tailed, and a threshold of $<0.05$ was considered for statistically significant associations.

\section{Patient and public involvement}

Patients and/or public were not involved in the design or conduct of this study.

\section{RESULTS}

Ultra-processed foods account for $56.8 \%$ of total energy intake and $64.7 \%$ of total free sugars in the UK diet. Unprocessed or minimally processed foods and processed culinary ingredients represented an additional $34.3 \%$ of total energy intake and $23.8 \%$ of free sugars, and processed foods the remaining $8.8 \%$ of total energy intake and $11.5 \%$ of free sugars. Ultra-processed foods accounted for a higher percentage of total energy intake among children $(63.5 \%)$ and adolescents $(68 \%)$. The average UK daily intake of free sugars was $12.4 \%$ (SE 0.1) of total energy intake and $61.3 \%$ of British exceeded the recommended limit of $10 \%$ energy from free sugars. This proportion was even higher among children $(74.9 \%)$ and adolescents $(82.9 \%)$ (table 1$)$.

No significant interaction was observed between the exposure and the survey year for the total energy intake from free sugars (unprocessed or minimally processed foods+processed culinary ingredients: $\mathrm{p}=0.254$; processed foods: $\mathrm{p}=0.538$; ultra-processed foods: $\mathrm{p}=0.137$ ) nor for the prevalence of excessive intake of free sugars (unprocessed or minimally processed foods+processed culinary ingredients: $p=0.609$; processed foods: $p=0.262$; ultra-processed foods: $\mathrm{p}=0.258)$. Even so, we included variable survey year (1-6) in the adjusted model.

Indicators of the dietary content in free sugars according to quintiles of the dietary contribution of NOVA food groups stratified by age groups are shown in tables $2-5$ ( $1.5-10$ years, $11-18$ years, $19-64$ years and $\geq 64$ years, respectively). The dietary contents of free sugars increased linearly across quintiles of ultra-processed food consumption for children (from $10.4 \%$ in the lowest quintile to $15.3 \%$ in the highest quintile), adolescents (from $12.7 \%$ to $17.4 \%$, respectively) and adults (from $9.6 \%$ to $15.2 \%$, respectively), whereas the increase for elderly was not significant (from $10.6 \%$ to $11.7 \%$, respectively). The prevalence of excessive free sugar intake also increased linearly across quintiles of ultra-processed food consumption for children, adolescents and adults. Children in the highest quintiles of ultra-processed food consumption had a prevalence of excessive free sugar intake $60 \%$ higher (PRadj 1.6; 95\% CI 1.3 to 1.9 ) than those in the lowest quintile group. The same trend was observed for adolescents (PRadj 1.6; 95\% CI 1.2 to 1.9) and adults (PRadj 1.7; 95\% CI 1.5 to 1. 9). Although no linear trend was found between quintiles of ultra-processed food consumption and excessive free sugar intake among elderly $(p>0.05)$, the fourth quintile group had a prevalence of excessive free sugar intake $35 \%$ higher (PRadj 1.3; 95\% CI 1.1 to 1.7) than those in the lowest quintile group.

Opposite trends were observed for the group of unprocessed or minimally processed foods and processed culinary ingredients, where the prevalence of excessive free sugar intake decreased from the first to the last quintile of these food groups in all age groups. The prevalence of excessive free sugar intake also decreased from the first to the last quintile of processed foods, but only in adolescents and adults.

Sensitivity analysis performed by considering complete cases only indicated that the results of the multiple imputations did not differ significantly from the complete case analysis (data not shown).

In our counterfactual scenarios, we calculated the percentage of excessive free sugar intake avoided if the consumption of ultra-processed foods and table sugar was zero (figure 1). We estimated that about $47 \%$ of the prevalence of excessive free sugar intake in the UK population could be potentially avoided if the consumption of ultra-processed foods was eliminated. Eliminating table sugar could potentially avoid $9.4 \%$ of the prevalence of excessive free sugar intake. This greater reduction in the percentage of excessive free sugar intake due to elimination of ultra-processed foods, relative to table sugar, was observed in all age groups, except in the elderly group where both scenarios had similar impacts on total free sugar intake. For the more feasible scenario, we found a similar trend where a greater reduction in the percentage of excessive free sugar intake due to a $50 \%$ reduction of ultra-processed foods, relative to table sugar, was observed in all age groups, except in the elderly group (see online supplementary figure S2).

\section{DISCUSSION}

In this large, nationally representative sample of the UK population, higher consumption of ultra-processed food was associated with greater dietary content of free sugars in children, adolescents and adults. Using theoretical minimum risk exposure level scenarios, we also showed that by eliminating ultra-processed food consumption, the prevalence of excessive free sugar intake $(10 \%$ or more of total energy intake) could be potentially reduced from $60 \%$ to $31 \%$. In children and adolescents, the potential reduction could be from $74 \%$ to $45 \%$ and from $83 \%$ to $53 \%$, respectively.

Our findings confirm an excessive consumption of free sugars in the UK diet ${ }^{13}$ and show that ultra-processed foods contributed to nearly $65 \%$ of all free sugars in all age groups and nearly $80 \%$ in children and adolescents. Unprocessed or minimally processed foods (mostly fresh juice) and processed culinary ingredients (mostly table sugar) contributed between $19 \%$ and $27 \%$ of the dietary content of free sugars, while processed foods provided the lowest contribution in all age groups. 


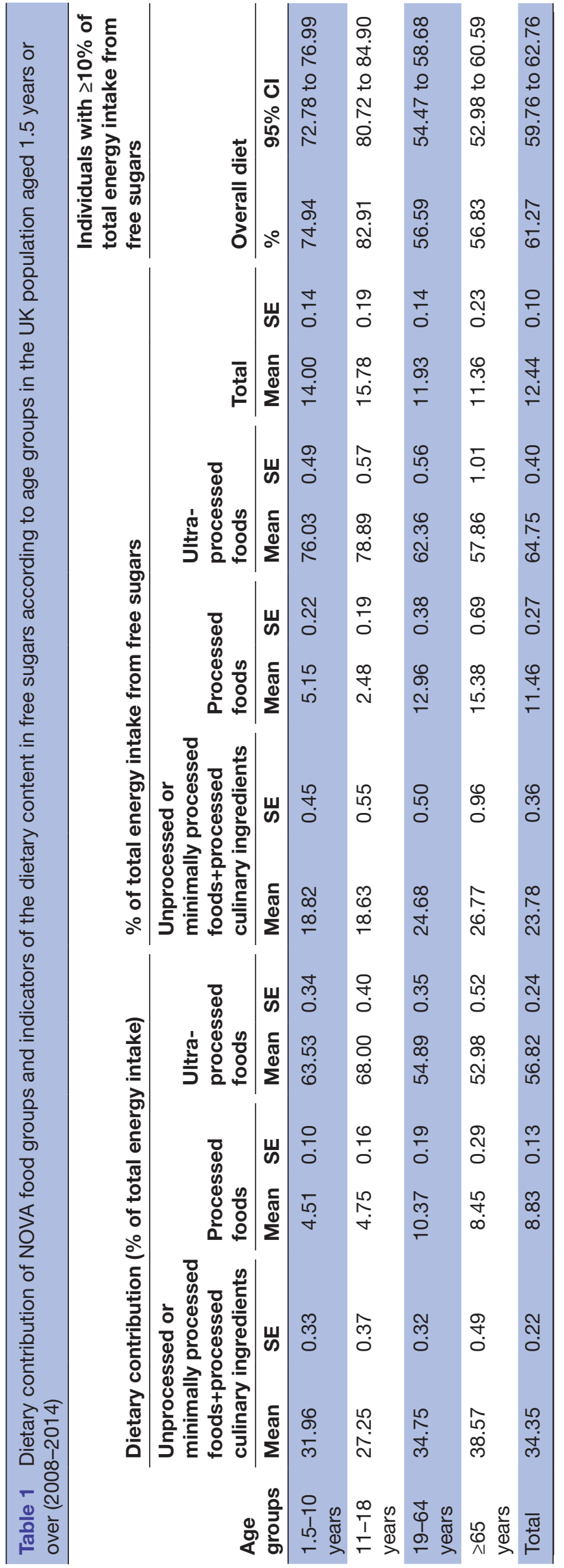




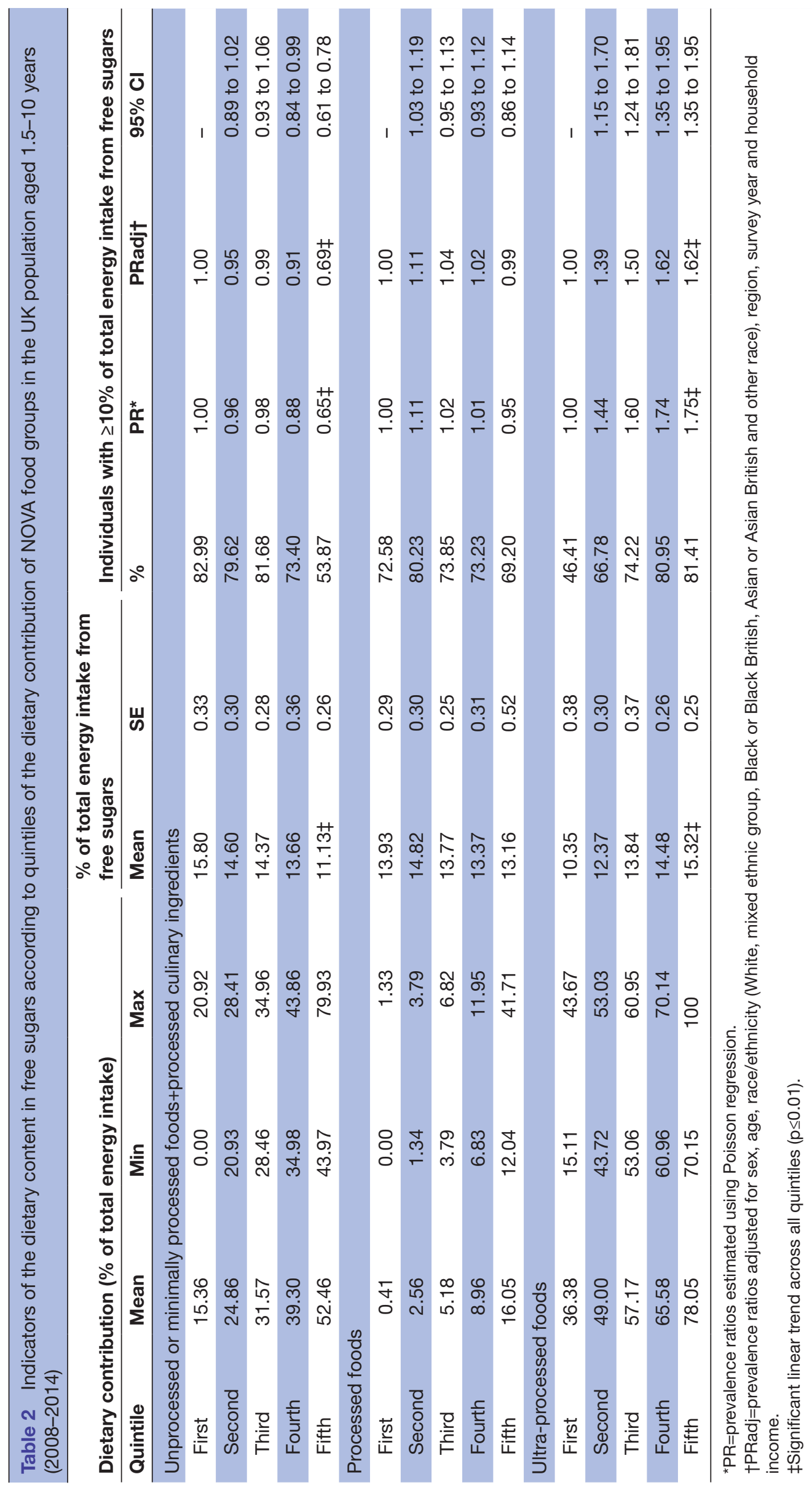




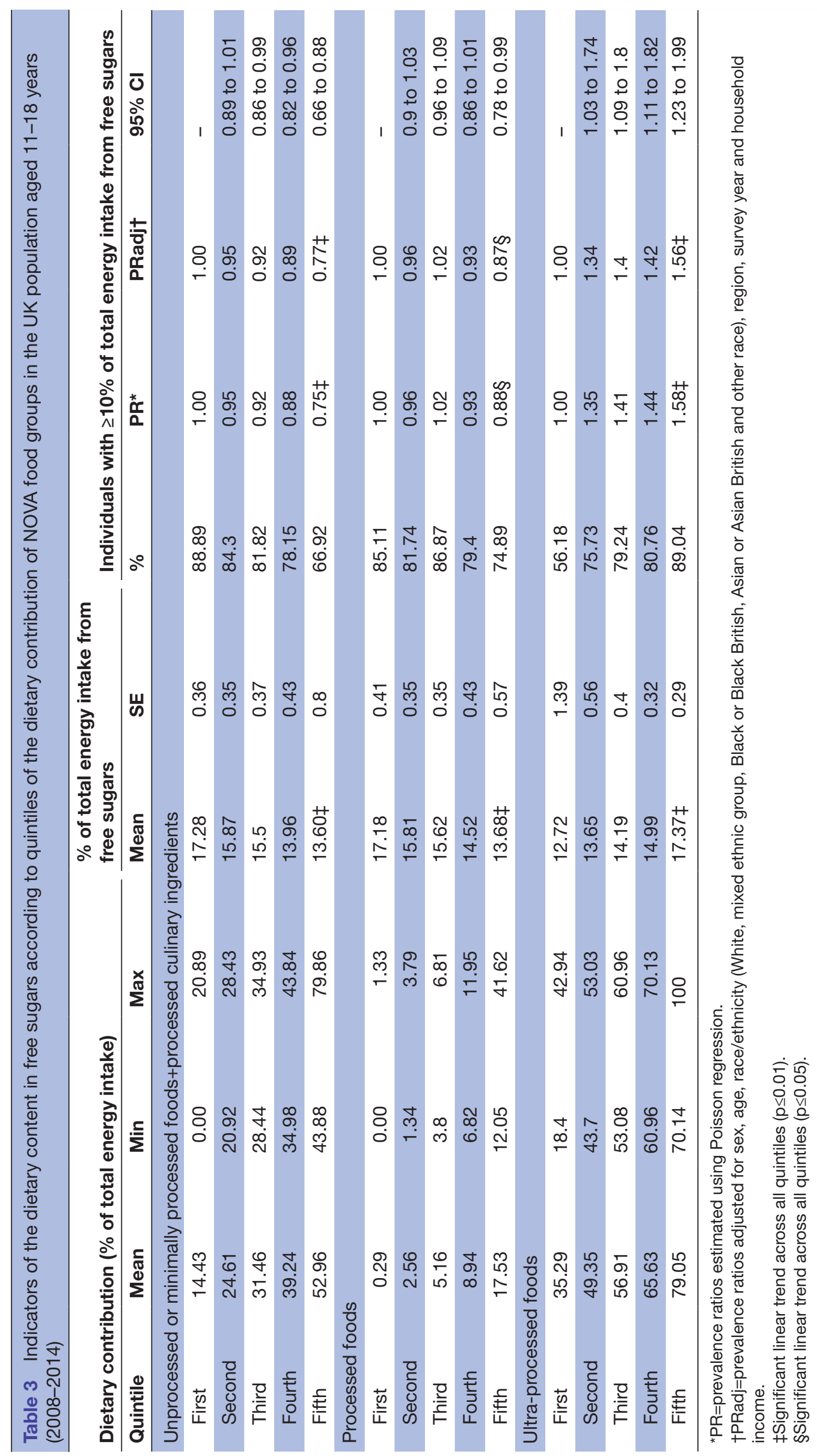


Table 4 Indicators of the dietary content in free sugars according to quintiles of the dietary contribution of NOVA food groups in the UK population aged 19-64 years (2008-2014)

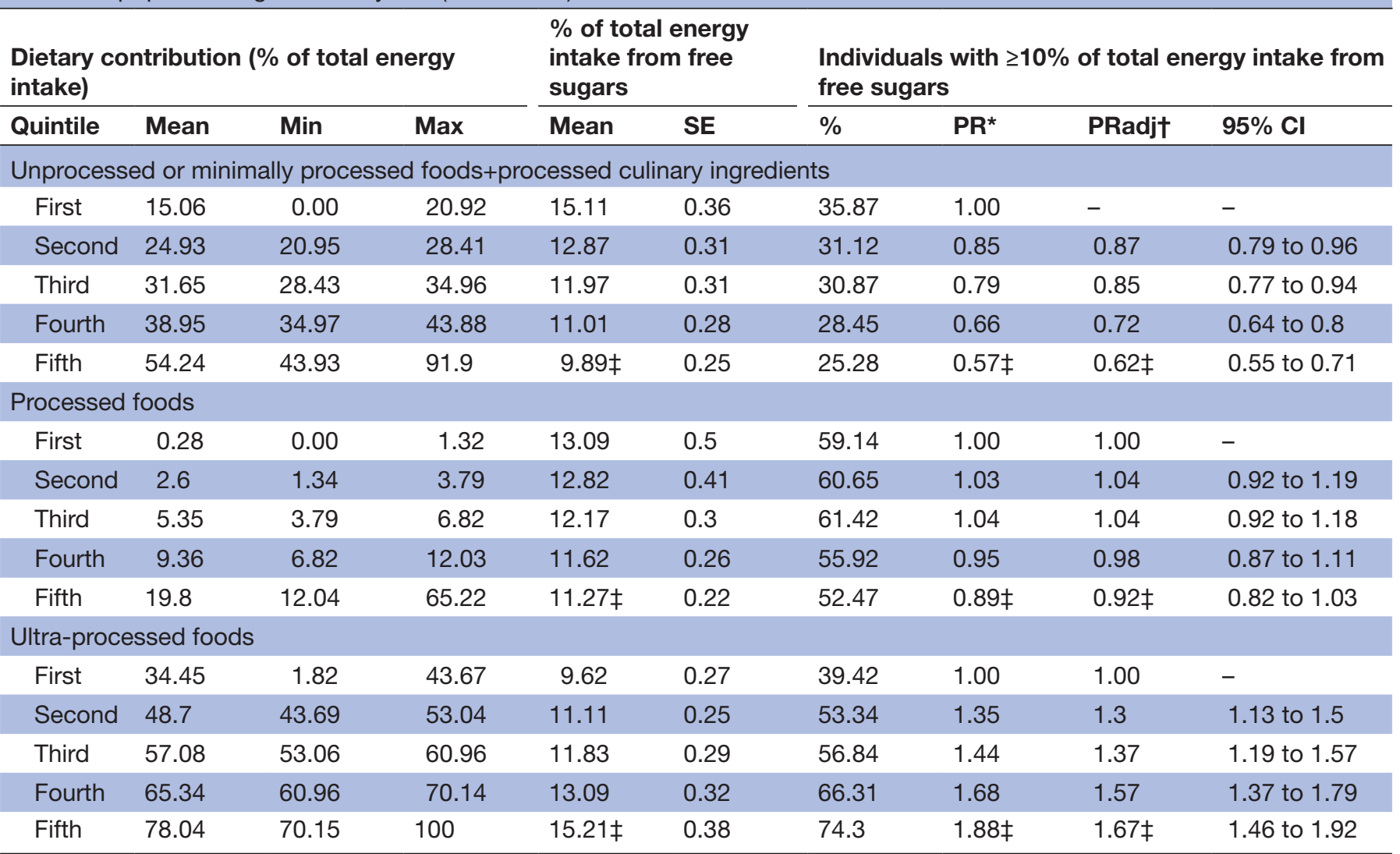

${ }^{*} \mathrm{PR}=$ prevalence ratios estimated using Poisson regression.

†PRadj=prevalence ratios adjusted for sex, age, race/ethnicity (White, mixed ethnic group, Black or Black British, Asian or Asian British and other race), region, survey year and household income.

$\ddagger$ Significant linear trend across all quintiles $(p \leq 0.01)$.

Our findings are similar to previous studies conducted in high-income and middle-income countries that have shown strong associations between the intake of ultra-processed foods and the dietary content of free sugars. ${ }^{8-11} \mathrm{~A}$ previous study conducted in Chile similarly showed that the association between ultra-processed food consumption and the dietary content of added sugars is more pronounced among children and adolescents. ${ }^{12}$ In our study, there was no linear association between ultra-processed food consumption and dietary content of free sugars among the elderly. Although the prevalence of excessive free sugar intake was higher in the fourth with regard to the first quintile of ultra-processed food consumption, the prevalence in the highest quintile group was not different from the first. A possible explanation for this finding could be changes in the composition of different types of ultra-processed across quintiles in the elderly. Actually, while in the overall population, ultra-processed sweetened products such as soft/fruit drinks, confectionery, milk-based drinks and biscuits monotonically increased across quintiles (from 18\% to $23 \%$ of the total calories from ultra-processed foods), in the elderly a drop in consumption was observed between the fourth and fifth quintiles (from $18 \%$ to $15 \%$ ) (data not shown).

There is strong evidence that the high consumption of free sugars contributes to excess obesity, type 2 diabetes, dyslipidaemia, hypertension and coronary heart disease. ${ }^{2-4}$ Consequently, most dietary recommendations now advise limiting free sugar intake, but more focused efforts are needed to put this recommendation into practice. Changing personal behaviour and choice alone is not an effective or realistic option as our findings confirm that the majority of free sugar is added to food before it is marketed and sold. Voluntary agreements between industry and government have been shown repeatedly to be ineffective in improving public health. ${ }^{24}$ This is confirmed by recent UK experience where the early stages of the government's sugar reduction programme, which challenged the food industry to voluntarily cut sugar in some products, have produced only slow progress towards proposed targets. ${ }^{25}$ Thus, more drastic measures that change the availability, price and marketing of these products are necessary.

The analyses presented here suggest that actions to reduce the consumption of ultra-processed foods often 
Table 5 Indicators of the dietary content in free sugars according to quintiles of the dietary contribution of NOVA food groups in the UK population aged 65 years or over (2008-2014)

\begin{tabular}{|c|c|c|c|c|c|c|c|c|c|}
\hline \multicolumn{3}{|c|}{$\begin{array}{l}\text { Dietary contribution ( } \% \text { of total } \\
\text { energy intake) }\end{array}$} & \multicolumn{3}{|c|}{$\begin{array}{l}\% \text { of total energy intake from free } \\
\text { sugars }\end{array}$} & \multicolumn{4}{|c|}{$\begin{array}{l}\text { Individuals with } \geq 10 \% \text { of total energy intake from } \\
\text { free sugars }\end{array}$} \\
\hline Quintile & Mean & Min & Max & Mean & SE & $\%$ & $\mathbf{P R}^{*}$ & PRadj† & $95 \% \mathrm{Cl}$ \\
\hline First & 16.63 & 6.34 & 20.82 & 11.67 & 0.87 & 56.16 & 1.00 & 1.00 & - \\
\hline Second & 25.04 & 20.95 & 28.36 & 12.83 & 0.61 & 67.39 & 1.2 & 1.19 & 0.9 to 1.57 \\
\hline Fifth & 52.26 & 43.89 & 78.36 & 10.7 & 0.42 & 50.94 & $0.91 \ddagger$ & $0.91 \ddagger$ & 0.69 to 1.21 \\
\hline \multicolumn{10}{|c|}{ Processed foods } \\
\hline First & 0.38 & 0.00 & 1.32 & & 0.72 & 43.52 & 1.00 & 1.00 & - \\
\hline Second & 2.42 & 1.34 & 3.78 & 9.7 & 0.56 & 64.3 & 1.48 & 1.49 & 1.14 to 1.96 \\
\hline \multicolumn{10}{|c|}{ Ultra-processed foods } \\
\hline First & 35.98 & 7.79 & 43.69 & 10.63 & 0.49 & 47.63 & 1.00 & 1.00 & - \\
\hline Second & 48.67 & 43.74 & 53.02 & 11.3 & 0.48 & 58.67 & 1.23 & 1.2 & 0.97 to 1.47 \\
\hline Third & 56.97 & 53.05 & 60.91 & 11.61 & 0.45 & 59.89 & 1.26 & 1.21 & 0.98 to 1.5 \\
\hline Fourth & 64.99 & 61.01 & 70.08 & 12.01 & 0.54 & 65.53 & 1.38 & 1.35 & 1.09 to 1.66 \\
\hline Fifth & 75.66 & 70.17 & 92.3 & 11.67 & 0.7 & 53.75 & 1.13 & 1.06 & 0.81 to 1.4 \\
\hline
\end{tabular}

${ }^{*} \mathrm{PR}=$ prevalence ratios estimated using Poisson regression.

†PRadj=prevalence ratios adjusted for sex, age, race/ethnicity (White, mixed ethnic group, Black or Black British, Asian or Asian British and other race), region, survey year and household income.

$\ddagger$ Significant linear trend across all quintiles $(p \leq 0.05)$.

Percentage of excessive free sugar intake that would be avoided under two counterfactual scenarios regarding the consumption of the main dietary sources of free sugar. UK population aged 1.5 years or over (2008-14).

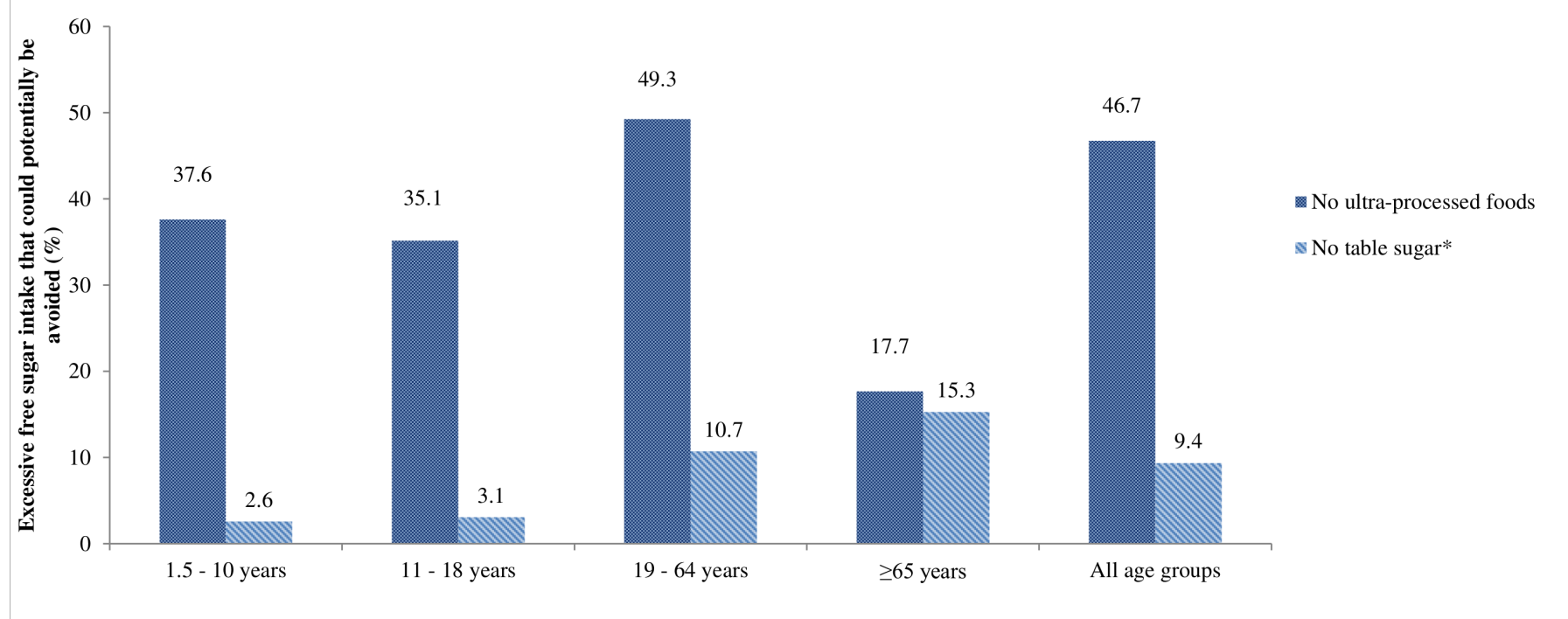

*Including honey, molasses, maple syrup (100\%).

Figure 1 Percentage of excessive free sugar intake that would be avoided under two counterfactual scenarios regarding the consumption of the main dietary sources of free sugar. UK population aged 1.5 years or over (2008-2014). 
rich in free sugars could lead to larger public health benefits. Policies concerning the use of fiscal measures to reduce intake of free sugars and improve diet quality should consider extending beyond artificially sweetened beverages to include the main driver of excessive free sugar intake, including dairy drinks, cakes, biscuits and confectionery. ${ }^{13}$

To our knowledge, this is the first study to examine the association between consumption of ultra-processed foods, as defined per NOVA, ${ }^{6}$ and dietary content of free sugar in different age groups of the UK population. The use of NOVA is a key strength of the study as it classified foods by their level of processing level using standardised and objective criteria. NOVA has been recognised as a valid tool for public health and nutrition research and policy by the Food and Agricultural Organisation of the United Nations $^{26}$ and the Pan American Health Organisation. ${ }^{27}$ In addition, we used data from the NDNS-a large and nationally representative sample of the UK population, applying weighting to reduce any sampling and non-response bias. Unlike household budget data, food diaries employed in the NDNS take food wastage into account, include food eaten out of home and do not assume that all individuals within a household consume the same diet. Importantly, the dietary data also allowed for the disaggregation of dishes into their constituents and classification of the underlying ingredients, which enabled the calculation of more precise estimates of intakes of each NOVA group and reduced misclassification.

Potential limitations should be considered. The dietary data we used were self-reported and may be subject to misclassification. A constant limitation of dietary assessment methods is under-reporting of some foods (particularly unhealthy foods), though food diaries are recognised to be one of the most comprehensive methods for assessing dietary intake. Possible under-reporting of unhealthy foods may lead to an underestimation of the dietary contribution of ultra-processed foods and the overall intake of free sugars but may less likely affect the association between these variables. Nevertheless, accurate and valid NDNS data were achieved through optimal methods for collecting dietary intake, ${ }^{28}$ which helped to minimise missing information. NDNS collects limited information indicative of food processing (eg, place of meals and product brands), which may lead to misclassification of food items. This bias is more likely for a small number of specific food items such as pizza where there is insufficient information for classification purposes (see online supplementary table S2). In those cases, the most frequently consumed alternative (culinary preparation or manufactured product) was chosen. Finally, our theoretical minimum risk exposure models estimate the potential impact of eliminating each of the main sources of free sugars on excessive free sugar intake, ignoring substitutions that may occur in the consumption of other foods. Although our findings suggest that greater reduction in excessive free sugar intake could be achieved by eliminating ultra-processed food consumption, guidance to the public about reducing the consumption of table sugar remains an important component of any public health guidance.

\section{Conclusion}

Almost half of excessive intake of free sugars in the UK can be attributed to ultra-processed foods. Policies to reduce sugar consumption should focus on minimising consumption of ultra-processed foods and replacing them with unprocessed or minimally processed food alternatives. The study adds to a growing body of evidence that ultra-processed foods are a major contributor to growth of diet-related non-communicable diseases globally.

\section{Author affiliations}

${ }^{1}$ Departamento de Nutrição, Universidade de Sao Paulo, Sao Paulo, Brazil

${ }^{2}$ Núcleo de Pesquisas Epidemiológicas em Nutrição e Saúde, Universidade de Sao Paulo, Sao Paulo, Brazil

${ }^{3}$ Departamento de Políticas Públicas e Saúde Coletiva, Universidade Federal de Sao Paulo, Sao Paulo, Brazil

${ }^{4}$ Escola Paulista de Medicina, Departamento de Medicina Preventiva, Universidade Federal de São Paulo, Sao Paulo, Brazil

${ }^{5}$ Public Health Policy Evaluation Unit, School of Public Health, Imperial College London, London, UK

${ }^{6}$ Departamento de Medicina Preventiva, Faculdade de Medicina, Universidade de Sao Paulo, Sao Paulo, Brazil

Contributors CAM, EMS, FR, MLdCL and RBL designed the research. FR and RBL undertook data management and analysis. CAM, CM, EMS, FR, LFMdR, MLdCL and RBL interpreted the data. FR wrote the first draft of the manuscript. All authors read, edited and approved the final manuscript.

Funding This work was supported by the Fundação de Amparo à Pesquisa do Estado de São Paulo (FAPESP), grant numbers 2015/14900-9, 2016/14302-7 (FR is a beneficiary of a postdoctoral fellowship) and 2014/25614-4 (LFMR is a beneficiary of a doctoral fellowship).

Disclaimer FAPESP had no role in the design, analysis or writing of this manuscript.

Competing interests None declared.

Patient consent for publication Not required.

Ethics approval All relevant research ethics and governance committees approved the survey.

Provenance and peer review Not commissioned; externally peer reviewed.

Data availability statement Computerized raw data files and documentation from this survey were obtained under license from the U.K. Data Archive (http:// www.esds.ac.uk). Details of how food item classification was accomplished are further explained in previously published papers (Rauber et al, Nutrients. 2018 - see Supplementary Table S1 http://www.mdpi.com/2072-6643/10/5/587/s1).

Open access This is an open access article distributed in accordance with the Creative Commons Attribution Non Commercial (CC BY-NC 4.0) license, which permits others to distribute, remix, adapt, build upon this work non-commercially, and license their derivative works on different terms, provided the original work is properly cited, appropriate credit is given, any changes made indicated, and the use is non-commercial. See: http://creativecommons.org/licenses/by-nc/4.0/.

ORCID iD

Fernanda Rauber http://orcid.org/0000-0001-9693-7954

\section{REFERENCES}

1 Scientific Advisory Committee on Nutrition. SACN's sugars and health recommendations: why 5\%. London, UK: Scientific Advisory Committee on Nutrition, Department of Health, 2015.

2 Te Morenga LA, Howatson AJ, Jones RM, et al. Dietary sugars and cardiometabolic risk: systematic review and meta-analyses of 
randomized controlled trials of the effects on blood pressure and lipids. Am J Clin Nutr 2014;100:65-79.

3 Scientific Advisory Committee on Nutrition. Carbohydrates and health report. London, UK: Scientific Advisory Committee on Nutrition, Department of Health, 2015.

4 Te Morenga L, Mallard S, Mann J. Dietary sugars and body weight: systematic review and meta-analyses of randomised controlled trials and cohort studies. BMJ 2012;346:e7492.

5 World Health Organization. Sugars intake for adults and children. Geneva, Switzerland: World Health Organization, 2015.

6 Monteiro CA, Cannon G, Moubarac J-C, et al. The un decade of nutrition, the nova food classification and the trouble with ultraprocessing. Public Health Nutr 2018;21:5-17.

7 Martínez Steele E, Popkin BM, Swinburn B, et al. The share of ultraprocessed foods and the overall nutritional quality of diets in the US: evidence from a nationally representative cross-sectional study. Popul Health Metr 2017;15:6.

8 Moubarac J-C, Batal M, Louzada ML, et al. Consumption of ultra-processed foods predicts diet quality in Canada. Appetite 2017;108:512-20.

9 Rauber F, da Costa Louzada ML, Steele EM, et al. Ultra-Processed food consumption and chronic non-communicable DiseasesRelated dietary nutrient profile in the UK (2008-2014). Nutrients 2018;10:E587.

10 Martínez Steele E, Baraldi LG, Louzada MLdaC, et al. Ultraprocessed foods and added sugars in the US diet: evidence from a nationally representative cross-sectional study. BMJ Open 2016;6:e009892.

11 Louzada MLdaC, Ricardo CZ, Steele EM, et al. The share of ultraprocessed foods determines the overall nutritional quality of diets in Brazil. Public Health Nutr 2018;21:94-102.

12 Cediel G, Reyes M, da Costa Louzada ML, et al. Ultra-processed foods and added sugars in the Chilean diet (2010). Public Health Nutr 2018;21:125-33.

13 Public Health England. National diet and nutrition survey results from years 7 and 8 (combined) of the rolling programme (2014/2015 to 2015/2016). London, UK: Public Health England, 2018.

14 Department of Health and Social Care: Global Public Health Directorate:Obesity, Food and Nutrition. Childhood obesity: a plan for action, chapter 2. London, UK: Department of Health and Social Care, 2018

15 Public Health England. National diet and nutrition survey results from years 1, 2, 3 and 4 (combined) of the rolling programme (2008/20092011/2012). London, UK: Public Health England, 2014.
16 Fitt E, Cole D, Ziauddeen N, et al. DINO (Diet In Nutrients Out) - an integrated dietary assessment system. Public Health Nutr 2015;18:234-41.

17 Public Health England. McCance and Widdowson's the composition of foods integrated dataset 2015. London, UK: Public Health England, 2015.

18 Monteiro CA, Cannon G, Levy RB, et al. The StAR shines bright. World Nutrition 2016;7:28-38.

19 Fitt E, Mak TN, Stephen AM, et al. Disaggregating composite food codes in the UK national diet and nutrition survey food composition databank. Eur J Clin Nutr 2010;64(Suppl 3):S32-6.

20 White IR, Royston P, Wood AM. Multiple imputation using chained equations: issues and guidance for practice. Stat Med 2011;30:377-99.

21 Nielsen SJ, Adair L. An alternative to dietary data exclusions. J Am Diet Assoc 2007;107:792-9.

22 Steenland K, Armstrong B. An overview of methods for calculating the burden of disease due to specific risk factors. Epidemiology 2006;17:512-9.

23 Rezende LFMde, Eluf-Neto J. Population attributable fraction: planning of diseases prevention actions in Brazil. Rev Saude Publica 2016;50:S0034-89102016000100601.

24 Moodie R, Stuckler D, Monteiro C, et al. Profits and pandemics: prevention of harmful effects of tobacco, alcohol, and ultraprocessed food and drink industries. Lancet 2013:381:670-9.

25 Public Health England. First measure of industry progress to cut sugar unveiled [press release]. London, UK: Public Health England, 2018.

26 Food and Agriculture Organization of the United Nations. Guidelines on the collection of information on food processing through food consumption surveys. Rome, Italy: Food and Agriculture Organization of the United Nations, 2015.

27 Pan American Health Organization. Ultra-processed food and drink products in Latin America: trends, impact on obesity, policy implications. Washington, DC: Pan American Health Organization, 2015.

28 Public Health England. Dietary data collection and editing. in national diet and nutrition survey. results from years 1-4 (combined) of the rolling programme (2008/2009-2011/2012. London, UK: Public Health England, 2014Available online:. https://www.gov.uk/ government/statistics/national-diet- and-nutrition-survey-resultsfrom-years-1-to-4-combined-of-the-rolling-programme-for-2008and-2009-to-2011-and-2012. (accessed on 15 January 2018). 